\title{
Pesquisa translacional do ensino superior no campo das ciências da saúde
}

\author{
Raphael Lopes Olegário ${ }^{1}$ \\ Pedro Victor Nogueira de Sousa ${ }^{2}$ \\ Silvia Maria Aparecida Vitorino ${ }^{3}$
}

Resumo A pesquisa translacional surge em um contexto de superespecialização das atividades de pesquisa científica, que trazem a necessidade de maior comunicação entre as áreas básica e clínica. $\mathrm{O}$ presente artigo procura elucidar a importância do incentivo à pesquisa proposta por parte do docente no ensino superior no campo das ciências da saúde com intuito de demonstrar a sua eficácia na formação profissional. Neste sentido, a mesma assume relevância preponderante para a formação acadêmica de graduandos e pós-graduandos da área da saúde. O objetivo dessa investigação foi mostrar por meio de uma revisão bibliográfica da literatura científica vigente, a importância do incentivo à pesquisa translacional nos cursos de ensino superior por parte do docente da área da saúde e os principais impactos na formação profissional. Utilizou-se como metodologia, uma investigação exploratória, por meio de uma pesquisa bibliográfica a partir de material já elaborado, constituído de artigos científicos e livros especializados. Como resultados, demonstrou-se que o incentivo à investigação translacional é a forma mais rápida para que a informação científica seja transmitida tanto para a investigação clínica quanto para a prática assistencial dos profissionais. Conclui-se que o incentivo a pesquisa translacional se

\footnotetext{
${ }^{1}$ Graduado em Educação Física. Universidade de Brasília rlounb@ gmail.com

${ }^{2}$ Graduado em Educação Física. Universidade de Brasília. Pedro_vnsouza@ hotmail.com

${ }^{3}$ Mestre em Tecnologia de Informação. Universidade de Federal de Uberlândia. silviavitorino2006@ yahoo.com.br
} 
caracteriza como essencial para prática profissional e este deve estar vinculado a prática pedagógica dos docentes na formação inicial ou continuada.

Palavras-chave: Pesquisa Translacional. Ensino. Medicina Translacional.

\title{
Translational reserch in higher education in the field of health sciences
}

\begin{abstract}
Translational research arises in a context of super-specialization of scientific research activities, which bring the need for greater communication between the basic and clinical areas. This article seeks to elucidate the importance of the research incentive proposed by the teacher in higher education in the field of health sciences in order to demonstrate their effectiveness in professional training. In this sense, it assumes preponderant relevance for the academic training of undergraduate and graduate students in the health area. The objective of this research was to show, through a bibliographical review of current scientific literature, the importance of the incentive to translational research in higher education courses by the health professors and the main impacts on professional training. An exploratory investigation was used as a methodology, through a bibliographical research from material already elaborated, consisting of scientific articles and specialized books. As results, it has been demonstrated that the incentive to translational research is the fastest way for scientific information to be transmitted both for clinical research and for the practice of care professionals. It is concluded that the incentive to translational research is characterized as essential for professional practice and this should be linked to the pedagogical practice of teachers in initial or continuing training.
\end{abstract}

Key words: Translational Research. Teaching. Translational Medicine.

\section{INTRODUÇÃO}

Com a necessidade de intervenção médica de forma mais efetiva e com maior inovação procedimental surge à medicina translacional com o objetivo de transferir os conhecimentos laboratoriais para a prática clínica do dia-a-dia (SCHMITTIDIEL et al., 2010). A pesquisa translacional pode ser definida como sendo toda a pesquisa com moldes iniciais nas ciências básicas e conclusão na aplicação prática do conhecimento. $\mathrm{Na}$ atualidade, a pesquisa translacional se divide em dois campos bem definidos: um relativo à pesquisa laboratorial e outro na pesquisa clínica, entretanto, em muitos casos não há vinculação entre os dois segmentos e, consequentemente, o conhecimento produzido pela pesquisa laboratorial não é bem aproveitado para propósitos de aplicação na prática clínica (FELDMAN, 2008).

A referida pesquisa possui uma característica de estudos já estabelecidos na literatura cientifica, como por exemplo, estudos clínicos e pesquisa aplicada, porém hoje em dia a pesquisa translacional visa passar a pratica laboratorial para a pesquisa clinica com seres humanos visando uma prática melhor (WEHLING, 2006; PUBMED, 2012). 
A investigação translacional teve como aliado o instituto nacional de câncer dos estados unidos (NCI) em 1992, que teve papel importante com incentivo a pesquisas e apoio financeiro para que estas fossem realizadas (GUIMARÃES, 2013). A pesquisa no âmbito translacional é uma ferramenta que pode ser utilizada para estreitar a relação entre descobertas científicas e ambiente prático, ou seja, minimizar a barreira existente entre meio científico e a prática profissional (SILVA, 2014). As ciências médicas possuem uma enorme dificuldade em solucionar esse conflito entre ciência e a prática, pesquisas laboratoriais e meio profissional, entre outras circunstancias, com isso a pesquisa translacional vem como forma de conhecimento para suprir ou buscar o entendimento de como quebrar essa barreira (WEHLING, 2010). Ao se considerar a relevância da pesquisa translacional no campo das ciências da saúde, é válido mencionar a urgente necessidade de identificar e solucionar problemas que minam a transferência efetiva do avanço científico em conhecimento útil e aplicado a determinado segmento. Este novo campo de investigação surge em um contexto de superespecialização das atividades de pesquisa científica, que trazem a necessidade de maior comunicação entre as áreas básica e clínica (WEHLING, 2010).

O presente artigo procura elucidar a importância do incentivo à pesquisa translacional por parte do docente do ensino superior no campo das ciências da saúde com intuito de demonstrar a sua eficácia na formação do profissional. Neste sentido, a referida pesquisa assume relevância preponderante para a formação acadêmica de graduandos e pós-graduandos da área da saúde. Dessa maneira, há que se considerar que o perfil do novo aluno no contexto globalizado, frente aos avanços tecnológicos da atualidade, requer dos professores e pesquisadores novas habilidades e competências que exigem a formação de um profissional mais crítico, autônomo e reflexivo.

O objetivo dessa investigação é mostrar por meio de uma revisão bibliográfica da literatura científica vigente, a importância do incentivo a pesquisa translacional nos cursos de ensino superior por parte do docente da área da saúde e os principais impactos na formação profissional. Pretende-se discutir a respeito das diferentes formas de conhecimento e evidenciar contribuições da pesquisa na formação dos futuros profissionais nos diversos segmentos de atuação. Para contemplar esses objetivos, adota-se a pesquisa bibliográfica em bases de dados especializadas das ciências da saúde e livros acadêmicos que abordem a temática. 
REVISTAELETRÔNICA DE GRADUAÇÃO

EPÓS-GRADUAÇĀO EM EDUCAÇĀO

REJ/UFG

\section{METODOLOGIA}

Foi realizado um estudo exploratório, por meio de uma pesquisa bibliográfica, que, em concordância com GIL (2008), "é desenvolvida a partir de material já elaborado, constituído de artigos científicos e livros especializados".

\subsection{Fontes}

Artigos científicos a respeito da temática foram acessados nas bases de dados: Scientific Electronic Library Online (Scielo), Biblioteca Virtual em Saúde (BVS) e Google Acadêmico. Os seguintes descritores foram utilizados, em língua portuguesa e inglesa, em concordância com os Descritores em Ciências da Saúde (DeCS) e Medical Subject Headings (MeSH): "translational medical research"; "applied research"; "translational science" e "translational medicine".

Livros especializados foram consultados e selecionados considerando-se a inserção das informações nos campos de pesquisa científica em saúde, práticas de ensino em saúde e medicina translacional.

\subsection{Coleta de dados}

A coleta de dados seguiu a seguinte sequência:

a) Leitura exploratória de todo o material pré-selecionado;

b) Leitura seletiva;

c) Registro das informações relevantes oriundas das fontes consultadas.

\subsection{Análise dos dados}

Nesta etapa foi realizada uma leitura de forma analítica com objetivo de ordenar as informações contidas nas diferentes fontes, de forma que estas possibilitassem a obtenção de dados relevantes a respeito do incentivo à pesquisa translacional do ensino superior no campo das ciências da saúde. 
A leitura analítica permite a identificação de termos e conceitos importantes para a investigação de determinado assunto, dessa maneira, conceitos e informações relevantes são extraídas da literatura e auxiliam na elaboração do texto formal.

O conhecimento científico obtido no processo metodológico tem como finalidade, na maioria das vezes, explicar e discutir um fenômeno baseado na verificação de uma ou mais hipóteses. Sendo assim, está diretamente vinculado a questões específicas na qual trata de explicá-las e relacioná-las com outros fatos (GALLIANO, 1986).

\subsection{Discussão dos resultados}

Informações relevantes oriundas das etapas anteriores foram analisadas e discutidas a partir do referencial teórico relativo à temática do estudo e registradas em software de texto.

\section{DESENVOLVIMENTO}

Segundo Baptista (2014) a investigação translacional é a forma mais rápida para que a informação científica seja transmitida tanto para a investigação clínica quanto para a prática assistencial. Isso significa uma transferência efetiva dos novos conhecimentos, mecanismos e técnicas em prol da saúde da população, colaborando, de forma inequívoca, para a tomada de decisões médicas e políticas. Também existe a noção que a pesquisa translacional transita em torno de processos lógicos que vão desde os estudos focados em chegar a uma nova fórmula, elemento molecular, etc. até de fato a aplicação dessa descoberta em estudos clínicos (CURRY, 2008; COLLER e CALLIF 2009; COLLINS, 2011).

A pesquisa translacional surge para tentar aproximar o pesquisador dos campos de prática. Embora o termo seja bastante recente, a noção de "transferência de resultados de pesquisa" não é. Esta vem sendo discutida desde as décadas de 1970 e 1980, nos Estados Unidos e também no Brasil (CASTRO et al, 1985).

O uso de novos recursos científicos aplicados na prática profissional no campo da saúde equivale a uma forma eficaz de geração e aplicação do conhecimento em moldes teóricos e práticos. O incentivo a investigação translacional nos cursos de formação inicial e continuada permite ao aluno a análise ampla do aspecto trabalhado no atual ou futuro campo de atuação 
profissional, visto que há necessidade de correlação dos conhecimentos oriundos de diversos segmentos para compreensão do todo.

Na perspectiva de Schwartz et al. (2017), a pesquisa translacional tem sido considerada em dois campos. A primeira, há transmissão de novos conhecimentos produzidos em ambiente laboratorial vinculados às ciências básicas referente aos mecanismos de produção de doença e resistência a drogas e quimioterápicos para o desenvolvimento de novos métodos de diagnóstico, tratamento e prevenção de diferentes patologias. A segunda, a investigação objetiva assegurar que os novos modelos de diagnóstico e tratamento cheguem aos pacientes ou às populações para as quais foram desenhados e que sejam aplicados de forma correta e com uma relação custo e benefício aceitável.

Ao articular ambos os campos de forma integrada, com aplicabilidade em situações da prática profissional de indivíduos em formação permite-se a assimilação de fatores importantes para análise de diversas situações, problemas comuns em diversos ambientes. A pesquisa científica significa alguma forma produtiva de conhecimento sobre determinado assunto através de diferentes métodos e estratégias fundamentadas. É relevante pontuar que os resultados das pesquisas devem superar as especulações do senso comum e permitir ao profissional a completa compreensão de todas as variações dos campos de estudos (SOARES JR, 2011).

A investigação translacional se caracteriza por um processo dual, já que os conhecimentos perpassam das ciências básicas para ciência clínica, e da clínica para a saúde dos pacientes e saúde pública. Em adição, é bidirecional, pois o fluxo de informações migra do laboratório à clínica e da clínica retorna ao laboratório (SÁENZ, 2011; LOPES-JUNIOR, 2015).

O incentivo à pesquisa científica, nos moldes de investigações translacionais devem ser apresentadas pelo docente de forma que o aluno compreenda a aplicabilidade desse conhecimento em sua rotina profissional. De acordo com Piaget (1990) o "ato de aprender, pela sua complexidade, exige um estudo que ultrapassa as raias da cognição, se encaminha para o afetivo/emocional, mergulha no social e se expande através do cultural". Nesse sentido, cabe ao professor auxiliar o aluno ao longo de seu desenvolvimento, de forma que este se torne apto a compreender a significação desse conhecimento para a sua formação. 
Percebe-se a importância de pesquisas que possam investigar melhores meios de se alcançar a reorganização do sistema de saúde, considerando às demandas médicas, de pesquisadores, gestores e pacientes, com intuito de produzir novos conhecimentos e estes se tornarem produtos que vão propiciar melhoria da saúde da população, sejam com um novo diagnóstico ou novos tratamentos terapêuticos, ou com ações que visem à promoção da saúde da população (ELIAS, 2012).

Ao relatar o processo de incentivo à pesquisa por meio da didática do professor, Ausubel (1980) afirma que trabalhar para que os estudantes aprendam significativamente a demanda e refletir sobre a essência da Aprendizagem Significativa, colaborar para que esses estudantes exercitem as interações mentais necessárias entre aquilo que já sabem e o novo conhecimento que lhes é apresentado, resgatando saberes prévios que, pouco utilizados no processo de aprendizagem, não fazem sentido para eles. Ou seja, a inserção de novos conhecimentos vinculados à pesquisas translacionais devem estar interligadas a conhecimentos prévios oriundos das vivências passadas dos alunos, seja em disciplinas, estágios ou atuações em ambiente profissional.

Como afirma Macedo (1994), o incentivo e aprendizagem dependem do desenvolvimento cognitivo, mas também depende da relação que o indivíduo constrói e mantém com o seu cotidiano, evidenciando, assim, as influências que as pessoas têm como mediadoras desse processo. Portanto, o processo de aprendizagem demanda múltiplos fatores que influenciaram na maneira pela qual o aluno compreenderá o conteúdo, assim como, a forma pela qual utilizará em ambiente profissional, podendo correlacionar a teoria com a prática.

Para Kolb (1974), o processo de aprendizagem se constitui no conhecimento vivenciado por meio da experiência. Para este autor, o processo pelo qual as pessoas aprendem podem ser sintetizados em um modelo vivencial de um ciclo quadrifásico, composto de quatro tipos de habilidades consolidadas (Figura 1): experiência concreta, observação reflexiva, conceituação abstrata e experimentação ativa. 


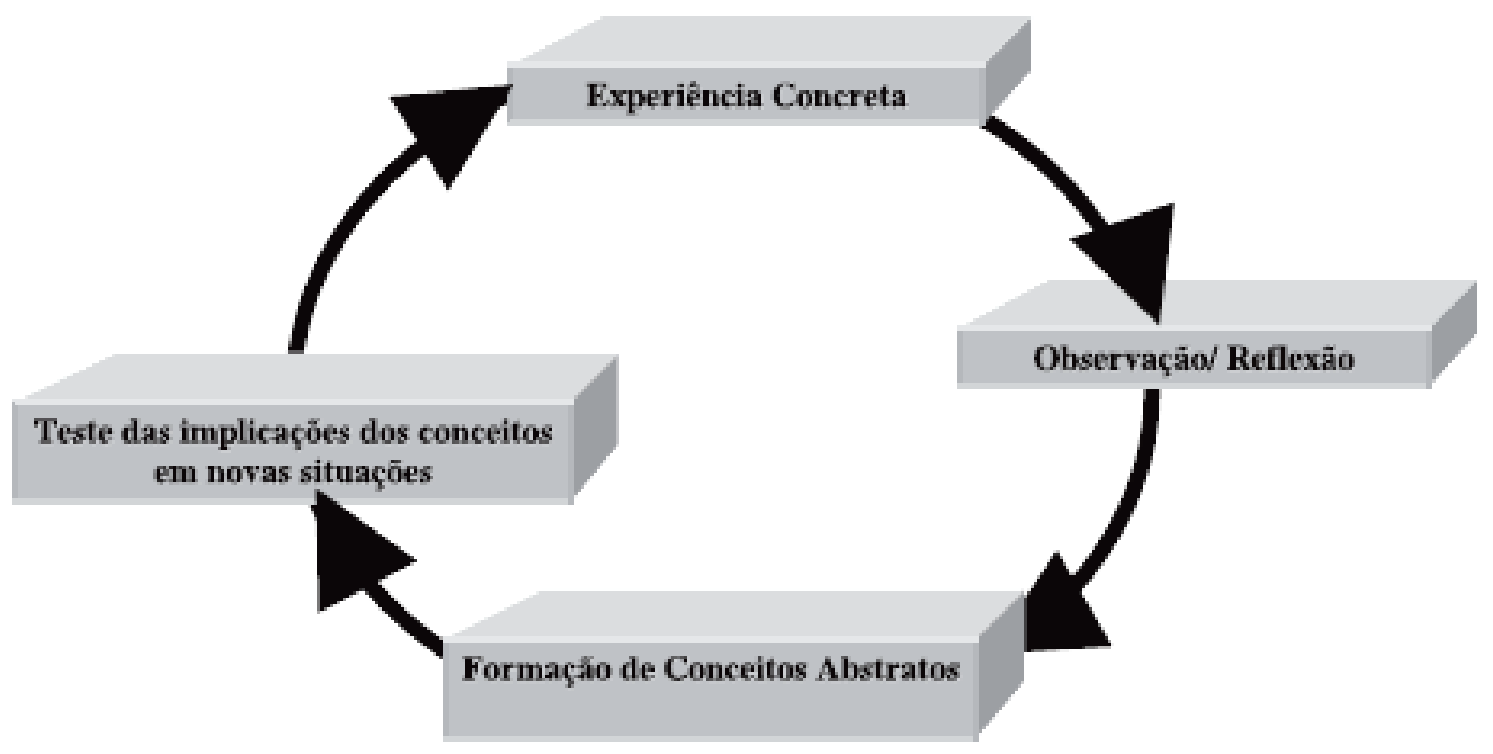

Fonte: Kolb, D. A., \& Fry, R. E. (1974). Toward an applied theory of experiential learning. MIT Alfred P. Sloan School of Management.

Em adição, Libâneo (1998) afirma que o professor medeia à relação ativa do aluno com a matéria, inclusive com os conteúdos próprios de sua disciplina, mas considerando o conhecimento, a experiência e o significado que o aluno traz à sala de aula, seu potencial cognitivo, sua capacidade e interesse, seu procedimento de pensar, seu modo de trabalhar. Desta maneira, o incentivo à pesquisa translacional deve estar situado de forma que o conhecimento prévio do aluno seja utilizado ao longo de todo o processo de aprendizagem.

\section{CONCLUSÃO}

O incentivo à pesquisa translacional configura-se como essencial para prática profissional e esta deve estar vinculada à prática pedagógica dos docentes na formação inicial ou continuada. As contribuições para prática profissional são relevantes, visto que o campo de investigação translacional procura correlacionar às intervenções clínicas e práticas. No intuito de proporcionar a formação completa do aluno, cabe ao docente desenvolver estratégias para a 
utilização de conhecimentos prévios dos alunos e contextualização com a pesquisa científica no âmbito da referida pesquisa.

\section{REFERÊNCIAS}

AUSUBEL, D. P.; NOVAK J. D., HANESIAN, H. Psicologia educacional. $2^{\text {a }}$. ed. Rio de Janeiro: Interamericana, 1980.

BAPTISTA, A.S.; MORELl, C.M.; SOZZI DE MORAIS, I. H. Proposta Conceitual de telessaúde no modelo de pesquisa translacional. Rev. Saúde Pública. v. 48. n. 2. p. 347-56. 2014.

CASTRO, I. B.; MIRANDA C. M. L., RODRIGUES A. P., SILVIA, M.J.. Dificuldades na incorporação dos resultados de pesquisa na prática da enfermagem. In: Anais do $4^{\circ}$ Seminário Nacional, Pesquisa em Enfermagem, 1985; São Paulo, Brasil. São Paulo: ABEn; 1985. p.193242.

COLLER, B. S., CALIFF, R. M. Traversing the valley of death: a guide to assessing prospects for translational success. Science Translational Medicine, v. 1, n. 10, p. 10, 2009.

COLLINS, F. S. Reengineering Translational Science: The Time Is Right. Science Translational Medicine, v. 3, n. 90, 2011.

CURRY, S. H. Translational science: Past, present, and future. Biotechniques, 44. 2008.

ELIAS, F. T. S. Revisões sistemáticas da literatura e o potencial ganho em eficiência no Sistema Único de Saúde. Tese (Doutorado) - Universidade Federal de São Paulo. São Paulo: 2012.

FELDMAN, A. Does Academic Culture Support Translational Research? CTS: Clinical and Translational Science. v. 48. n. 2. p.87-8. 2008.

GALLIANO, Alfredo Guilherme. O método científico: teoria e prática. São Paulo: Harbra, 1986.

GIL, A. C. Métodos e técnicas de pesquisa social. 6. ed. São Paulo: Atlas, 2008. 
GUIMARÃES, R. Translational research: an interpretation. Ciencia \& Saude Coletiva, v. 18, n. 6, p. 1731-1744, 2013.

KOLD, D. A., \& Fry, R. E. (1974). Toward an applied theory of experiential learning. MIT Alfred P. Sloan School of Management.

LIBÂNEO, J. C. Adeusprofessor, adeus professora?Novas exigências educacionais e profissão docente. São Paulo: Cortez, 1998. p. 29.

LOPES-JÚNIOR, L. C. Pesquisa translacional e a enfermagem: da bancada de laboratório à beira do leito. Journal of Nursing UFPE online. v. 9. n.12. p. 1-2. 2015.

MACEDO, L. Ensaios Construtivistas. São Paulo: Casa do Psicólogo Livraria e Editora Ltda., 1994, p.134.

PIAGET, J. Seis estudos de Psicologia. Rio de Janeiro: Forense Universitária Ltda, 1990, p.12.

PUBMED. Translational research Disponível em:

< https://www.ncbi.nlm.nih.gov/mesh/?term=translational+research >. Acessado em 21 maio 2019.

SÁENZ C, et al. Medicina translacional: del laboratório a la clínica y de la clínica hasta la acción. Gastroenterología Latinoamericana. v.22.n.3. p.263-4, 2011.

SCHMITTDIEL JA, GRUMBACH K, SHELBY JV. System-based participatory research in health care: an approach for sustainable translational research and quality improvement. Ann Fam Med. v. 8 n. 3, p. 256-9, 2010.

SCHWARTZ J, MACOMBER C. So, You Think You Have an Idea: A Practical Risk Reduction-Conceptual Model for Academic Translational Research. Bioengineering. v.4n.2p. 29, 2017.

SILVA, E. R. P. Duality in a volcanic temple: a critical assessment of management's neverending crisis (Doctoral dissertation, Universidade Federal do Rio de Janeiro). 2014.

SOARES J.R., N. E. A pesquisa no currículo de um curso de formação inicial dos professores de Educação Física. Revista Pensar a Prática, Goiânia, v. 14, n. 1, p. 1-12.2011. 
WEHLING, M. Principles of translational science in medicine: from bench to bedside. Cambridge; New York: Cambridge University Press, 2010.

WEHLING, M. Translational medicine: can it really facilitate the transition of research "from bench to bedside"? European Journal of Clinical Pharmacology, v. 62, n. 2, p. 91-95. 2006. 